\title{
RESIDUOS: \\ UNA REELABORACIÓN CONCEPTUAL
}

\author{
Alma Paola Tietbohl Urrego* \\ Julio César Rivera Rodríguez \\ Licenciados en Química. Universidad Pedagógica Nacional
}

\begin{abstract}
The authors present in this article a summary of the research carried out at Jorge Eliécer Gaitán, a public School in Bogotá, during 1999, based on the design and implementation of a pedagogic and didactic strategy, for tenth level students focused on chan ge in conceptual, attitudinal, methodological, axiological and esthetical change of (ECMMAs) at the residuals.
\end{abstract}

\section{RESUMEN}

En este artículo, los autores presentan los resultados obtenidos en la investigación realizada durante el año de 1999, en el Colegio Distrital Jorge Eliecer Gaitán, a través del diseño y ejecución de una Estrategia Pedagógica y Didáctica para estudiantes de grado décimo (10) centrada en el cambio Conceptual, Metodológico, Actitudinal, Axiológico y Estético (ECMAAS) sobre los residuos.

\section{INTRODUCCIÓN}

Los seres humanos han aprendido a transformar los compuestos naturales y a producir nuevas sustancias que no existían en el medio. A medida que se fueron procesando, surgió un excedente no útil dentro del proceso, los residuos. Produciendo de esta manera, un gran amontonamiento inútil, que en su mayoría no se pueden "Introducir en los ciclos biogeoquímicos de la naturaleza”. (Cuadernos de educación ambiental 1994).

Abandonar los residuos sin medir las consecuencias que sobre el sistema tierra, esta actitud genera, no constituye una alternativa positiva para el mantenimiento y mejoramiento de la calidad de vida, y obedece a pensar, que por parte de los individuos no existe una elaboración racional de lo que son dichos materiales. El fin inmediato es desaparecerlos, sin considerar los efectos que estos provocan sobre el entorno, generando así, una alteración permanente y un desequilibrio constante del sistema tierra. Teniendo en cuenta lo dicho anteriormente, se puede afirmar que ha predominado una idea lineal y reduccionista de los residuos en cuanto a su manejo, como un proceso repetitivo del actuar del individuo, el cual sólo tiende a botarlos, sin construir en algún momento, una idea conceptual y divergente de ellos, que permita romper el esquema tradicionista que alberga su cotidiano vivir puesto que no son beneficiosos para el en ninguna medida.

Por ello, comprender qué son los residuos y cómo estos se han convertido en un problema, ayudaría a entender que así como todas las personas tienen que ver con su mal manejo, también todos son responsables y deben contribuir a la solución de los

\footnotetext{
*Estudiante de Maestría en Docencia de la Química. Universidad Pedagógica Nacional.
} 
problemas y de las causas que los generan. Es decir, teniendo una comprensión y una reelaboración del concepto, se contribuiría ha ampliar el horizonte de trabajo e investigación en otras disciplinas, pues al determinar que los residuos son susceptibles de tratamientos industriales, los especialistas, por ejemplo, en energías alternativas en siderurgia y metalmecánica, en papeles y cartones, y obviamente los especialistas en Química Ambiental en aspectos tales como lixiviados, efluentes y aguas residuales, ahorrarían bastante energía, dinero y aprovecharían gran cantidad de material que consideran éstos como basura.

En este sentido, para los investigadores fue de gran interés auscultar en la población objeto de estudio (Colegio Distrital Jorge Eliecer Gaitán - Grado 1003), si las causas de este comportamiento eran el producto de las estructuras de pensamiento que manejaban sobre los residuos y lograr a través de una estrategia pedagógico-didáctica una transformación intelectual de los sujetos en cuanto a lo conceptual, lo metodológico, lo actitudinal y lo axiológico (ECMAAs). De esta manera, la reelaboración de lo que son los residuos se abordó desde la Teoría de la Complejidad expuesta dentro del programa de investigación Representación y Conceptos Científicos (1994), el cual es dirigido por los profesores Royman Pérez Miranda y Rómulo Gallego Badillo, del departamento de Química, de la Universidad Pedagógica Nacional.

Por tal razón, para esta investigación se admite la aproximación del aprendizaje total, en la cual el aprendizaje se asume como cambio conceptual, metodológico, actitudinal, axiológico, donde cada una de estas categorías se codefinen y codeterminan, de tal manera que es imposible interrogarse sobre cada una de ellas sin tener en cuenta las otras (Gallego, et al.,1997). Además, se tomo como base de trabajo el concepto de residuos, ya que no existen estudios realizados en el campo educativo que pretendan modificar las estructuras de pensamiento sobre estos materiales, pues los estudios encontrados son más de carácter técnico y contribuyen a tratamientos específicos sobre los mismos.

\section{MARCO CONCEPTUAL}

A finales de los setenta, aparece en el mundo científico la idea de la tierra como entidad compleja (Enciclopedia de pedagogía 1988), la cual comprende una enorme diversidad de componentes y de interacciones que constituyen un sistema capaz de autorregularse, y de mantener un entorno fisicoquímico óptimo para la vida. En esta medida, surge un factor importante bastante relacionado, dennotado, como la crisis del pensamiento filosófico y científico que da lugar al desarrollo de un nuevo paradigma: (definiéndose este como realizaciones cognitivas propias de cada individuo que durante cierto tiempo le proporcionan modelos de problemas y soluciones a diferentes situaciones) con la perspectiva compleja y ecológica del conocimiento, la cual se puede estudiar desde la interacción continua de sistemas. Por ejemplo, el universo se con figura como una arquitectura de sistemas que interactúan unos con otros, el ser humano se integra en un sistema socionatural, que a su vez es parte de un sistema planetario. 


\begin{tabular}{|c|c|}
\hline Perspectiva de la Simplificación & Perspectiva de la Complejidad \\
\hline $\begin{array}{l}\text { Los campos del saber se presentan } \\
\text { disjuntos, se concibe los residuos } \\
\text { como inútiles. }\end{array}$ & $\begin{array}{l}\text { Se busca la articulación de conceptos } \\
\text { y se conciben los residuos como útiles. }\end{array}$ \\
\hline $\begin{array}{c}\text { Enfoque analítico centrado en el estudio } \\
\text { de elementos aislados y modelos } \\
\text { precisos, } \\
\text { se toman los Residuos como un simple } \\
\text { concepto Aislado. }\end{array}$ & $\begin{array}{c}\text { Enfoque sistemático centrado en el estudio } \\
\text { de las interacciones entre los elementos, } \\
\text { se toma la basura, como un concepto } \\
\text { complejo que interactúa con su medio. }\end{array}$ \\
\hline $\begin{array}{c}\text { Visión estática basada en lo permanente, } \\
\text { y comportamientos previsibles, } \\
\text { los residuos permanecen estáticos } \\
\text { pues se entierran. }\end{array}$ & $\begin{array}{c}\text { Visión dinámica basada en } \\
\text { comportamientos } \\
\text { imprevisibles, se toman los residuos } \\
\text { como una manera útil de mantener } \\
\text { el equilibrio del planeta. }\end{array}$ \\
\hline $\begin{array}{l}\text { Causalidad lineal y eficiente, los residuos } \\
\text { se entierran y se descomponen. }\end{array}$ & $\begin{array}{l}\text { Causalidad circular, interacción, } \\
\text { los residuos tienen propósitos de utilidad. }\end{array}$ \\
\hline $\begin{array}{c}\text { Se trabaja con la perspectiva de residuo } \\
\text { como objeto. }\end{array}$ & $\begin{array}{c}\text { Se trabaja la implicación de los residuos } \\
\text { en el sistema tierra. }\end{array}$ \\
\hline
\end{tabular}

Desde una perspectiva compleja, la naturaleza no es una colección de entes separados sino un sistema de sistemas conectados e interdependientes, la cual se afecta por el sistema socionatural, donde el hombre a través del mal manejo e interpretación que le da a los residuos, a su vez lo desequilibra. De esta manera el concepto residuo debe ser complejo en el individuo, en la medida en que lo vea como un objeto útil, dándole una visión interactiva con el sistema socionatural y a la vez con el sistema tierra.

Teniendo en cuenta la anterior apreciación, se consideró necesario, para la presente investigación, tener claro lo que es entendido como posición constructivista. La principal postura de quienes trabajan bajo esta perspectiva epistemológica, es la de que los seres humanos elaboran suposiciones y creencias básicas acerca de la estructura y funcionamiento del mundo a partir de las cuales direccionan y elaboran unos espacios exponenciales con miras a construir un mundo para sí (Gallego, R. Pérez, M. 1994) las cuales organiza en estructuras Conceptuales, Metodológicas, Actitudinaíes y Axiológicas (ECMAAs), las cuales van vinculadas entre sí, lo actitudinal es actuacional y por tanto metodológico y conceptual, donde se lleva un proceso de construcción de significados formas de significar y de actuar, la transformación de una de estas ECMAAs se halla determinada por las modificaciones de las otras, introduciéndose en consecuencia el problema de la complejidad centrado en las interacciones significativas entre estas. No obstante, es necesario men-. cionar que la complejidad interna que tienen los individuos en sus estructuras mentales se hallan también en interacción con el entorno que lo rodea, de esta manera el aprendizaje como cambio en las ECMAAs no se puede ver desde la perspectiva lineal o desde la ontología parmenídea sino desde el punto de vista complejo heraclíteo, pues el aprendizaje se lleva a cabo a través de procesos activos de construcción y reconstrucción de significados, formas de significar y de actuar, que implican un desarrollo potencial de la actividad cognoscitiva del individuo. 
De esta manera, al trabajar con el concepto de residuos, debe surgir en los individuos una estructura es de significado y de significación, de tal manera que el sujeto desde ella le atribuye a lo nuevo un significado y es dicho significado, el que es incorporado a la estructura cognoscitiva como sistema de significación (Gallego, R. Pérez, R. 1997).

Dentro de esta perspectiva, se consideró necesario, para el desarrollo del concepto residuo, con la población objeto de estudio, la interacción entre el constructivismo y la teoría del Caos. El constructivismo, con la concepción de que los estudiantes realizan una actividad cognoscitiva respecto a los residuos, la cual se traduce en una información que permite interactuar con los diferentes miembros de la población objeto de estudio. Desde la teoría del caos, la actividad cognoscitiva no presenta una mecánica lineal, sino obedece a una constante evolución y cambio permanente de lo que son estos materiales, asumiéndolos con una visión holística la cual permite favorecer el sistema tierra.

\section{INTENCIONALIDADES}

Con este trabajo se pretendió:

- Establecer las posibles transformaciones de significados, formas de significar y de actuar que sobre el concepto residuo presenta la población objeto de estudio, a través de una estrategia pedagógica y didáctica diseñada para ello.

- Diseñar, desarrollar y validar algunos instrumentos que permitan identificar y caracterizar las representaciones que sobre el concepto de residuo poseen los estudiantes de la población objeto de estudio, antes y después de aplicada la estrategia pedagógico-didáctica diseñada para el efecto.

- A través de la estrategia pedagógico-didáctica diseñada, determinar como la población objeto de estudio, hace una construcción y reconstrucción del con-copto residuo.

\section{MARCO METODOLOGICO}

La investigación se efectúo con 36 estudiantes entre los 15 y 19 años de edad, de ambos géneros masculino y femenino, los cuales cursaban grado décimo en el colegio Distrital Jorge Eliecer Gaitán. La problemática radicó en auscultar las concepciones que de partida poseía la población objeto de estudio respecto del concepto residuo, de esta manera se recolecto información que identificaba las transformaciones operadas en las estructuras de pensamiento aludidas, en cuanto a la reelaboración del concepto Basura hacia el de residuo, entendido éste como un excedente aprovechable de la actividad humana, que en consecuencia puede ser fuente de riqueza y materia prima de otros procesos.

Se dio inicio a la estrategia pedagógica y didáctica con la aplicación de dos instrumentos (mapas conceptuales y prueba tipo likert), con el propósito de auscultar las ideas alternativas que poseía la población objeto de estudio, sobre el con-copto base de trabajo.

Luego del trabajo realizado anteriormente, se dio continuidad a la estrategia con el desarrollo de dos seminarios acerca de los residuos. El primero, es un trabajo de 
conceptualización acerca de: las capas concéntricas de la tierra, la importancia de la Biósfera, la diferenciación entre material orgánico e inorgánico, la diferencia entre basura y residuo, el reciclaje. El segundo, fue un trabajo sobre el manejo adecuado y buen tratamiento de los residuos, el cual abordó las siguientes temáticas: tratamiento adecuado de los residuos, efectos en el medio ambiente tales como: el efecto invernadero, disminución de la capa de ozono, lluvia ácida, erosión, salinización, acumulación de metales pesados y los efectos nocivos en el hombre. Se procedió a la aplicación del tercer instrumento que fue el cuestionario.

Finaliza a la estrategia pedagógico-didáctica aplicando de nuevo los instrumentos empleados al inicio, con el fin de ver si se produjo o no una transformación en las estructuras de pensamiento.

Los instrumentos empleados fueron:

- Mapas conceptuales, los cuales fueron empleados al inicio y finalización de la investigación, como mecanismo para establecer cambios progresivos en la construcción de significados, formas de significar y de actuar en los estudiantes de grado décimo.

- Pruebas tipo likert, para auscultar la interacción entre los componentes Conceptual, Metodológico, Actitudinal, Axiológico, Estético, y a su vez con el propósito de auscultar las ideas alternativas que posee la población objeto de estudio, sobre el concepto base de trabajo.

- Cuestionario de tres preguntas, para identificar las representaciones de los estudiantes en relación con los conceptos de Compuestos Orgánicos, Compuestos Inorgánicos y residuos.

\section{RESULTADOS}

De acuerdo a la investigación realizada con los estudiantes de grado décimo del Colegio Distrital Jorge Eliecer Gaitán, y la información obtenida respecto de sus representaciones, se tuvo en cuenta las explicaciones significativas o existencia de modelos coherentes, el uso del lenguaje propio y científico y la aproximación de significados frente a la temática planteada.

Al inicio del proceso, se encuentra que en las pruebas tipo likert aplicadas, predomina una posición por parte de la población objeto de estudio bastante sesgada y reduccionista donde existe además un rechazo permanente frente a la temática planteada, ya que la mayoría de estudiantes conciben a los residuos como material inútil y estorboso de los cuales no existe algún provecho. En relación con este instrumento, al finalizar el proceso, se encuentra que la mayoría de los estudiantes de la población objeto de estudio presentan una posición bastante diferente hacia los residuos, ya que los ven como material útil y aprovechable, mientras algunos de ellos, tienden a cambiar su posición frente a los residuos de una manera parcial, es decir, se presentan cambios en algunas de sus estructuras de pensamiento, también se encuentra, que un estudiante persiste con la concepción de material inútil y estorboso.

El análisis de los mapas conceptuales se realizó desde una perspectiva cualitativa, la construcción de los mismos, por parte de la población objeto de estudio presentó varias dificultades al inicio del proceso, ya que no se tenía una familiarización con dicho 
instrumento, y además, se manejaban arbitrariamente proposiciones incoherentes mediante un lenguaje cotidiano bastante desarticulado frente a la temática planteada, con carencia de relaciones cruzadas en los mismos. Al finalizar el proceso, se evidencia un cambio bastante significativo en la elaboración de los mapas conceptuales por parte de los estudiantes, ya que se presentan modelos explicativos y coherentes con relaciones cruzadas y jerarquización de conceptos. Además, la concepción final plasmada en dichas construcciones, presenta a los residuos como materiales útiles, transformables y reutilizables que pueden ser material aprovechable.

De otra forma, en los cuestionarios presentados por los estudiantes de grado décimo, el significado del concepto residuo durante el transcurso de la investigación es sinónimo de basura como un material inútil, el cual no se puede transformar. Posteriormente, se encuentra que la mayoría de los estudiantes consideran a los residuos como un materiales útiles, renovables y transformables, los cuales se pueden aprovechar, ya que, expresan sus ideas de una manera clara y coherente, con un uso de lenguaje científico, dando respuesta a las preguntas de dicho instrumento.

\section{CONCLUSIONES}

Las representaciones iniciales de la población objeto de estudio en relación con el concepto residuos, eran concepciones bastantes razas y dispersas cuyo significado se encontraba alejado del aceptado por los investigadores.

Los investigadores concluyen de este trabajo que el problema fundamental de los residuos, no es un problema técnico ni de tratamientos tecnológicos, es un problema educativo que obedece al cambio en las estructuras de pensamiento de los individuos, es decir, su solución está, en la modificación de las estructuras conceptuales, metodológicas, actitudinales, axiológicas, estéticas (ECMAAs) como se presentaron en los estudiantes del Colegio Distrital Jorge Eliecer Gaitán.

La estrategia pedagógico-didáctica empleada, efectuó cambios significativos en la mayoría de la población objeto de estudio, respecto de las residuos, pues al finalizar el proceso, se encontró que de acuerdo a la realización de esta, se 0pta por para ver estos materiales como fuente de riqueza útil y aprovechable, ya que modifican las formas de significar y de actuar, y además reelaboran y construyen una nueva forma de pensamiento acerca de los mismos.

A través del proceso realizado, se lograron comprobar las hipótesis planteadas de este proyecto de investigación, ya que de entrada se encontró que la población objeto de estudio presentaba la concepción de la basura como basura, y desarrollada la estrategia pedagógico-didáctica, los individuos modifican dicha concepción por la de un material útil y aprovechable.

Efectuado el trabajo realizado por los investigadores, se recolecto la información suficiente para solucionar la problemática planteada. Además, la información recopilada, muestra que a través de la estrategia aplicada, hubo manifestación de cambio en la población objeto de estudio respecto del concepto base de trabajo.

Este trabajo de investigación, con los estudiantes del Colegio Distrital Jorge Eliecer Gaitán, es el comienzo de una labor permanente y cambiante, pues no se puede continuar con la concepción de las basuras como materiales inútiles e inoficiosos en el 
sistema educativo y debe continuar, para así modificar el esquema predominante de las nuevas generaciones que hacen parte de la comunidad educativa.

\section{BIBLIOGRAFIA}

Gallego, R. y Pérez, R.1994. Corrientes constructivistas. Cooperativa editorial del Magisterio. 1997. El concepto de aprendizaje total. Estudios en pedagogía y didáctica. 1994. Representaciones y conceptos científicos. Programa de Investigación. Universidad Pedagógica Nacional. Departamento de Química. 1997. Un concepto de aprendizaje total. Una aproximación teórica. 2 (1).

Cuadernos de educación ambiental. Centre Unesco de Catalunya. Julio 1994.

Enciclopedia práctica de pedagogía. Técnicas pedagógicas. Editorial planeta. Vol. 5.1988. 